142. Richard Meyer und A. Jugilewitgoh: Ueber einige Fster der Phtalsăure und Tetraohlorphtelsăure.

[Mitgetheilt von Rich. Meyer]

(Eingegangen am 1. April).

Vor etwa 4 Jahren untersuchte ich die Einwirkung von Phtalylchlorid auf einige $\mathrm{Phenole}^{1}$ ) und erhielt dabei im Allgemeinen dio Phtalsãureester der letzteren.

Nur in einem Falle, nãmlich bei der Umsetzung mit $\beta$-Naphtol, vollzog sich noch eine Nebenreaction, welche zur Bildung von $\beta$-Naphtofluoran führte. Da den Estern der Phtalsäure wohl im Allgemeinen - in Rücksicht auf ihre Bildung aus phtalsaurem Silber und Halogenalkylen - symmetrische Structur zugeschrieben wird, dio Fluorane aber unzweifelhaft auf den unsymmetrischen Typus bezogen werden müssen, so regte diese Erfahrung von Neuem die Frage nach der Constitution und den Reactionsverhältnissen des Phtalylchlorides bezw. der Phtalsäureester an. In dieser Hinsicht ist es von besonderer Wichtigkeit, zu wissen, ob die aus phtalsaurem Silber und Halogenalkylen entstehenden Ester mit den durch Umsetzung von Phtalylchlorid mit Alkoholen resultirenden identisch oder isomer sind.

Zur Beurtheilung dieser Frage hat C. Graebe ${ }^{2}$ ) bereits einige experimentelle Beiträge geliefert, ohne jedoch ein entscheidendes Ergebniss zu erzielen. Auf meinen $W$ unsch hat deshalb Herr Stud. A. Jugilewitsch eine Reihe solcher Ester der Phtalsăure und Tetrachlorphtalsäure nach den beiden Methoden dargestellt, von denen erwartet werden konnte, dass ihre physikalischen Eigenschaften eine scharfe Vergleichung ermöglichen würden.

Die ersten Ergebnisse dieser Untersuchung sind schon ganz kurz angekündigt worden ${ }^{3}$ ). Die Versuche sind seither auf eine ganze Reihe von Estern ausgedehnt worden und sollen im Folgenden karz beschrieben werden. In allen untersuchten Fallen haben sich die nach beiden Methoden dargestellten Ester als durchaus identisch erwiesen.

\title{
Phtalsaurer Benzylester.
}

a) Aus phtalsaurem Silber und Benzyljod.

Nachdem ein Vorversuch gezeigt, dass Benzylchlorid mit phtalsaurem Silber nicht in Reaction gebracht werden kann, wurde die

1) Diese Berichte 26, 204.

2) Diese Berichte 16, 860; Ann. d. Chem. 838, 325.

3) Diese Berichte 88, 1577. Die an dieser Stelle angegebene Bildung eines gemischten Anhydrids der Phtalsăure und Benzoēsănre hat sich bei gonauerer Prüfung als irrig erwiesen. 
Umsetzung mittels des Jodids herbeigeführt. Reibt man die beiden Körper in berechneter Menge zusammen, so tritt Erwärmung ein; zur Vollendung der Reaction erwärmt man zweckmksaig noch 1 bis 2 Stunden auf dem Wasserbade, bis der Geruch nach Benzyljodid versehwunden ist. Durch Extrahiren mit Alkohol wird der Eeter von dem gleichzeitig gebildeten Jodsilber getrennt; ersterer krystallisirt dann beim Erkalten der alkobolischen Lösung in dicken Prismen, welche durch Umkrystallisiren aus Alkohol unter Anwendung von Thierkohle rein und farblos erhalten werden.

Analyse: Ber. făr $\mathrm{C}_{6} \mathrm{H}_{4}\left(\mathrm{COO} . \mathrm{CH}_{2} \mathrm{C}_{6} \mathrm{H}_{5}\right)_{2}$.

$$
\begin{aligned}
\text { Procente: } & \mathrm{C} 76.3, \quad \mathrm{H} 5.2 . \\
\text { Gef. } \gg & \rightarrow 76.9,76.3, \approx 5.5,5.8 .
\end{aligned}
$$

Der phtaleaure Benzylester schmilzt bei $42-43^{\circ}$. Er löst sich leicht in den meisten der üblichen Lösungsmittel, schwieriger aber in Weingeist, sehr wenig in Ligroïn und Petroleumbenzin, nicht merkbar in Wasser. Durch Kochen mit alkoholischem Kali wird er leicht verseift unter Bildung von Phtalsäure und Benzylalkohol (s. u.).

Wendet man das Benzyljodid in feuchtem Zustande an, 80 erhält man statt des neutralen Esters, oder neben demselben, den sauren phtalsauren Benzylester, welcher aus Alkohol in hübschen Prismen anschiesst. Es erwies sich zweckmăseig, ihn in verdünnter $\mathrm{Na-}$ tronlange zu lösen und mit Salzsäure wieder auszufällen; schliesslich warde er noch in Alkohol gelöst und mit Wasser wieder gefăllt. So gereinigt, schmilzt der Körper bei 102-1040. Er zeigt ähnliche Löolichkeitsverhältnisse wie der neutrale Ester, ist aber in Alkohol weit leichter löslich, als dieser. Die alkoholische Lösung färbt blaues, mit Wasser angefeuchtetes Lakmuspapier roth. Bei der Verseifung wurden auch in diesem Falle Phtalsäure und Benzylalkohol erbalten.

Analyse: Ber. für $\mathrm{C}_{6} \mathrm{H}_{6}<\mathrm{COO} . \mathrm{CH}_{2} . \mathrm{C}_{6} \mathrm{H}_{5}$.

$$
\begin{aligned}
& \text { Procente: C 70.31, H 4.69. } \\
& \text { Gef. \ 》70.12, 》 } 4.71 \text {. }
\end{aligned}
$$

b) Aus Phtalylchlorid und Benzylalkohol.

Man löst in Benzylalkohol die für die Bildung von Natrinmbenzylat berechnete Menge Natrium auf; nachdem alles gelöst und die Masse fest geworden, setzt man allmählich die theoretische Menge Phtalylchlorid hinzu. Die Reaction tritt Bchon bei gewöhnlicher Temperatur ein, man erhitzt aber schliesslich noch auf dem Wasserbade, bis der Geruch nach Phtalylchlorid verschwunden ist. Die Masee warde dann mit Sodalösung ${ }^{1}$ ) behandelt und mit Aether geschüttelt.

1) Ans dieser wurde durch Anshnern und Extrahiren mit Aother ein Gernisch von Phtaleăure und Benzoēsăure abgesohieden; letztere stammte offenbar aus dem verwendeten Benzylalkohol. 
Letzterer hinterliess ein gelbes Oel, welches der Destillation unterworfen wurde. Es ging zuerst Wasser über; dann zwischen 180 bis $210^{\circ}$ nnangegriffener Benzylalkohol und weiter zwischen 290-310 Phtalsăureanhydrid (welches wohl in dem angewandten Phtalylohlorid enthalten war). Da bei weiterem Erhitzen weisse Dampfe auftraten, welche auf Zersetzung hinwiesen, so wurde die Destillation abgebrochen. Der Kolbeninhalt erstarrte beim Erkalten und bestand der Hauptsache nach ans dem neutralen phtalsauren Benzylester. Derselbe wurde durch Umkrystallisiren aus Alkohol mit Thierkohle rein erhalten, und zeigte dann alle Eigenschaften des aus phtalsaurem Silber und Benzyljodid erhaltenen Präparats. Schmp. 42-43\%. Die Ausbeute betrug 70-80 pCt. der Theorie.

Analyse: Ber. für $\mathrm{C}_{6} \mathrm{H}_{4}\left(\mathrm{COO} . \mathrm{CH}_{2} \mathrm{C}_{6} \mathrm{H}_{5}\right)_{2}$.

$$
\begin{aligned}
& \text { Procente: C 76.30, H 5.20. } \\
& \text { Gef. " 》75.92, 76.27 }>5.5,5.29 \text {. }
\end{aligned}
$$

Der Ester wird durch Kochen mit alkoholischem Kali leicht verseift. Es scheiden sich schon in der Hitze Blättchen von phtalsaurem Kalium ab. Aus diesem wurde die Phtalsäure isolirt und in Form des Anhydrids durch Schmelzpunkt und Fluoresceïnreaction identificirt. Die von dem phtalsauren Kalium abgesaugte alkoholisch-alkalische Lösung wurde mit Wasser verdünnt und mit Aether extrahirt. Letzterer hinterliess beim Verdunsten den Benzylalkohol, welcher noch weiter durch Oxydation zu Benzaldehyd und Benzoësäure (durch Salpetersäure) charakterisirt wurde.

\section{Phtalsaurer $p$-Nitrobenzylester.}

a) Aus phtalsaurem Silber und $p$-Nitrobenzyljodid.

Ebenso wie Benzylchlorid, so reagirt auch $p$-Nitrobenzylchlorid nicht mit phtalsaurem Silber, weshalb die Darstellung des Esters mittels $p$-Nitrobenzyljodid bewirkt werden musste. Dasselbe wurde nach G. Ku mp ${ }^{1}$ ) durch Kochen von $p$-Nitrobenzylchlorid ${ }^{2}$ ) mit Jodkalium in alkoholischer Lösung bereitet. Es war nach mehrmaligem Umkrystallisiren aus Alkohol rein und zeigte den von $K u m p f$ angegebenen Schmp. $127^{\circ}$.

2 Mol. dieses Jodids wurden mit 1 Mol. phtalsaurem Silber 1 bis 2 Stunden auf dem Wasserbade erhitzt und die Masse dann mit Benzol extrahirt. Beim Erkalten der Lösung schied sich daraus der Ester in langen Nadeln ab, welche durch mehrmaliges Umkrystallisiren aus viel Benzol mit Thierkoble gereinigt wurden. Der Körper ist in

1) Ann. d. Chem. 224, 99.

2) Es wurde ein von der Firma Kalle \& Co. in Biebrich a. Rh. freundlichst zur Verfügung gestelltes Product verwendet, welches aber zuvor durch Umkrystallisiren gereinigt wurde. 
heissem Ligroïn, Eisessig, Benzol, Amylalkohol ziemlich leicht, sehr schwer löslich aber in Alkohol. Schmp. 154-1550.

Analyso: Ber. für $\mathrm{C}_{6} \mathrm{H}_{4}\left(\mathrm{COO} \text {. } \mathrm{CH}_{2} \mathrm{C}_{6} \mathrm{H}_{4} \text {. } \mathrm{NO}_{2}\right)_{2}$.

Procente: N 6.40.
Gef.

Bei dem Versuche, den Ester mit alkoholischem Kali zu verseifen, schied sich ein rothbrauner amorpher Körper ab, vermuthlich derselbe, welchen H. Klinge ${ }^{1}$ ) durch Einwirkung von Natriummethylat auf $p$-Nitrotoluol in methylalkoholischer Lösung erbalten hat. Er zeigte mit concentrirter Schwefelsăure die von Klinger angegebene blaue Färbung, wurde aber nicht weiter untersucht. Aus der mit Wasser verdünnten und von dem rothen Körper abfiltrirten Lösung konnte leicht Phtalsäure abgeschieden und als solche charakterisirt werden.

b) Aus Phtalylchlorid und $p$-Nitrobenzylalkohol.

Der erforderliche $p$-Nitrobenzylalkohol wurde aus einem technischen, zuvor durch Umkrystallisation gereinigten $p$-Nitrobenzaldehyd ${ }^{2}$ ) darch Einwirkang von 15-procentiger Natronlauge bereitet. (Es entstand auch hierbei eine gewisse Menge des Klinger'schen rothen Körpers). Der mehrmals umkrystallisirte, $p$-Nitrobenzylalkohol zeigte den richtigen Schmelzpunkt $93^{\circ}$.

Zur Darstellung des Esters konnte diesmal die Anwendung von Natrium umgangen werden. Es genügt, $p$-Nitrobenzylalkahol und Phtalylchlorid in berechneter Menge auf dem Wasserbade mit einander zu erwärmen. Bald beginnt eine starke HCl-Entwickelung und nach kurzer Zeit erstarrt die Masse. Man erwärmt noch bis zum Aufhören der $\mathrm{HCl}$-Entwickelung, behandelt dann mit kalter Natronlauge, filtrirt den Rückstand und trocknet ihn. Der Ester kann aus Amylalkohol, oder besser aus viel Benzol umkrystallisirt werden. Die Eigenschaften entsprechen den oben angegebenen; Schmelzpunkt 154 bis $155^{\circ}$.

Analyse: Ber. für $\mathrm{C}_{6} \mathrm{H}_{4}\left(\mathrm{COO}, \mathrm{CH}_{2}, \mathrm{C}_{6} \mathrm{H}_{4} \cdot \mathrm{NO}_{2}\right)_{2}$.

Procente: N 6.40.

Gef. \ "6.49.

Phtalsaurer Cetylester.

a) Aus phtalsaurem Silber und Cetyljodid.

Man bringt die berechneten Mengen der beiden Körper ') zusammen, worauf die Reaction schon in der Kălte beginnt; sie wird

1) Diese Berichete 15, 866; 16, 941.

7) Auch dieses Product verdanke ich der Gäte der Firma Kallo \& Co.

3) Das Cetyljodid war von Kahlbaum bezogen. 
dorch 1-2-stãndiges Erwärmen auf dem Wasserbade beendet. Der gebildete Ester wird dann mit Alkohol extrahirt, aus welchem er durch Umkrystallisiren mit Thierkohle in feinen Nadelchen erhalten wird. Er löst sich leicht in Aceton, Essigăther und Methylalkohol and schmilzt bei $42-43^{\circ}$ ).

Analyse: Ber. für $\mathrm{C}_{6} \mathrm{H}_{4}\left(\mathrm{COO}_{16} \mathrm{O}_{33}\right)$.

$$
\begin{aligned}
& \text { Procente: C 78.18, H 11.40. } \\
& \text { Gef. 》 》 78.69, 78.35, 》11.69, } 11.50 \text {. }
\end{aligned}
$$

Beim Verseifen mit alkoholischem Kali gab er Phtalsăure und Cetylalkohol, welch letzterer durch den Schmelzpunkt $49-50^{\circ}$ identificirt werden konnte.

$$
\text { b) Aus Phtalylchlorid und Cetylalkohol. }
$$

Man bereitet zunächst Natriumcetylat durch Eintragen der berechneten Menge Natrium in im Oelbade bei $110^{\circ}$ geschmolzenen Cetylalkohol ${ }^{2}$ ) und bringt dann allmählich die nöthige Menge Phtalylchlorid hinzu. Die Umsetzung wird durch Erwärmen auf dem Wasserbade bir zum Verschwinden des Geruchs nach Phtalylchlorid zu Ende geführt. Man versetzt dann mit verdünnter Natronlauge und nimmt mit Aether auf. Letzterer hinterlässt den phtalsauren Cetylester, welcher durch Umkrystallisiren aus Alkohol mit Thierkohle rein erhalten wird. Die Eigenschaften sind die oben angegebenen Schmelzpunkte $42-43^{\circ}$. Auch die Verseifung lieferte dieselben Ergebnisse.

Analyse: Ber. für $\mathrm{C}_{6} \mathrm{H}_{4}\left(\mathrm{COOC}_{16} \mathrm{H}_{33}\right)_{2}$.

$$
\begin{array}{cccc}
\text { Procente C } 78.18, & \text { H } 11.40 . \\
\text { Gef. } & \gg 78.30, &
\end{array}
$$

Tetrachlorphtalsaurer Benzylester.

a) Aus tetrachlorphtalsaurem Silber und Benzyljodid.

Als Ausgangsmaterial für die Darstellung dieser und der folgenden Verbindungen diente eine technische Tetrachlorphtalsäure der Badischen Anilin- und Soda-Fabrik, welche durch mebrmaliges Umkrystallisiren aus heissem Wasser gereinigt, den Schmp. 249-250 ${ }^{\circ}$ besass.

1 Mol. tetrachlorphtalsaures Silber und 2 Mol. Benzyljodid wurden vermischt. Die Reaction vollzieht sich freiwillig und unter Wärmeentwickelung; sie wird durch 2-3-stündiges Erwärmen auf dem W asserbade zu Ende gebracht. Der gebildete Ester wird der Reactionsmasse durch Extrahiren mit Weingeist oder Holzgeist entzogen und durch Umkrystallisiren aus letzterem in langen farblosen Nadeln erhalten. Schmelzpunkt $92-93^{\circ}$. Die Ausbeute war quantitativ.

1) Zufăllig genau übereinstimmend mit dem Benzylester.

7) Gleichfalls von Kahlbaum. 
Analyse: Ber. für $\mathrm{C}_{6} \mathrm{Cl}_{4}\left(\mathrm{COO} . \mathrm{CH}_{2} \mathrm{C}_{8} \mathrm{H}_{6}\right)$.

Procente: Cl 29.34.

Gef. $\gg \quad 29.43$.

Bei der Verseifung wurde', neben Benzylalkohol, Tetrachilorphtalsăare erhalten. Ersterer wurde wieder durch Ueberfūhren in Benzaldehyd und Benzoẽsäare charakterisirt; letztere gab beim Sublimiren das bei $243-245^{\circ}$ schmelzende Anhydrid und durch Zusammenschmelzen mit Resorcin die Fluoresceinnreaction.

b) Aus Tetrachlorphtalylchlorid und Benzylalkohol.

Das Chlorid der Tetrachlorphtalsăure wurde nach den Angaben von Graebe 1) dargestellt. Da die Tetrachłorphtalsăure sehr geneigt ist, saure Ester zu bilden, so warde, um dies zu vermeiden, besonders darauf geachtet, dsss der Benzylalkohol vollstăndig als Natrinmbenzylat in Reaction tritt. Von diesem wurde dann ein kleiner Ueberschuss angewendet. Man erwärmt das Gemisch anf dem Wasserbade bis zum Verschwinden des Chloridgeruchs und isolirt das Product wieder durch Behandeln mit verdünnter Natronlauge, Extrahiren mit Aether und Umkrystallisation aus Holzgeist. Der Ester wurde wieder in Nadeln vom Schmelzpunkt 92-930 erhalten. Bei der Verseifung resultirte auch in diesem Falle Tetrachlorphtalsăure and Benzylalkohol.

$$
\begin{array}{r}
\text { Analyse: Ber. für } \mathrm{C}_{6} \mathrm{Cl}_{4}\left(\mathrm{COO} . \mathrm{CH}_{2} \mathrm{C}_{6} \mathrm{H}_{3}\right)_{2} . \\
\text { Procente: } \mathrm{Cl} 29.34 . \\
\text { Gef. } \quad \text { \% } 29.58 .
\end{array}
$$

Wie schon bemerkt, entsteht, wenn neben Natriumbenzylat noch freier Benzylalkohol einwirkt, ausser dem neutralen auch eine gewisse Menge sauren Esters. Derselbe geht nebst freier Tetrachlorphtalsăure in das alkalische Waschwasser über. Er kann von der Säure durch seine Löslichkeit in Benzol getrennt werden; aus der Benzollösung wird er durch Petroleumbenzin gefällt. Nach mehrfacher Wiederholung der Operation war er rein. Er schmilzt bei $130-131^{\circ}$.

Analyse: Ber. für $\mathrm{C}_{6} \mathrm{Cl}_{4}<\mathrm{COO}$. $\mathrm{CH}_{2} \mathrm{C}_{6} \mathrm{H}_{5}$.

$$
\text { Procente: Cl 36.05. }
$$

$$
\text { Gef. » \36.55. }
$$

Tetrachlorphtalsaurer $p$-Nitrobenzylester.

a) Aus tetrachlorphtalsaurem Silber und $p$-Nitrobenzyljodid.

Beim Zusammenreiben eines dem theoretischen Verhältnisse entsprechenden Gemenges der beiden Körper zeigt sich die auffallende Erscheinung, dass dieselben schon bei gewöhnlicher Temperatur, wobei die Mischung fest und trocken ist, auf einander einwirken. Dies

1) Ann. d. Chem. 238, 328. 
giebt sich deutlich durch Gelbfärbung in Folge der Bildung von Jodsilber zu erkennen. Um die Reaction zu Ende zu fähren, erwärmt man noch eine Stunde auf dem Wasserbade, wobei aber auch keine Verflūssigung eintritt. ( $p$-Nitrobenzyljodid schmilzt bei $127^{\circ}$, der neu gebildete Ester bei $179-180^{\circ}$.) Trotzdem ist die Umsetzung eine vollständige. Der Ester wird von dem Jodsilber durch Extrahiren mit heissem Benzol getrennt, woraus er leicht rein erhalten werden kann. Er bildet dann lange Nadeln; Schmp. 179-180 . Bei der Verseifung warde neben Tetrachlorphtalsäure wieder der rothe (Klinger'sche?) Körper erbalten.

Analyso: Ber. für $\mathrm{C}_{6} \mathrm{Cl}_{4}\left(\mathrm{COO} . \mathrm{CH}_{3} \mathrm{C}_{6} \mathrm{H}_{4} \mathrm{NO}_{2}\right)_{2}$.

$$
\begin{aligned}
& \text { Procente: N 4.88, Cl 24.74. } \\
& \text { Gef. \ } \quad 5.45 \text {, } 24.77 \text {. }
\end{aligned}
$$

b) Aus Tetrachlorphtalylchlorid und $p$-Nitrobenzylalkohol.

Zur Darstellung von Natrium-p-Nitrobenzylat konnte man nicht den trockenen Alkohol anwenden, da Natrium ihn unter Feuererscheinung sofort verkohlt. Man musste ihn deshalb in einem gegen Natrium indifferenten Lösungsmittel auflösen, durfte jedoch die Lösung nicht zu verdünnt nehmen, da sonst die Reaction zu träge verläuft, oder ganz ausbleibt. Es wurde deshalb eine concentrirte Lösung in Benzol verwendet; letzteres war vorher mit Natrium getrocknet worden. In das so bereitete Alkoholat wurde die berechnete Menge Tetrachlorphtalylchlorid gebracht und 3 bis 4 Stunden auf dem Wasserbade erwärmt; darauf mit Natronlauge behandelt, mit Aether aufgenommen und aus Benzol umkrystallisirt. Die Eigenschaften des Esters stimmten vollkommen mit dem aus Silbersalz und Jodid erhaltenen. Schmp. $179-180^{\circ}$. Die Ausbeute war gering.

Analyse: Ber. für $\mathrm{C}_{6} \mathrm{Cl}_{4}\left(\mathrm{COO} . \mathrm{CH}_{2} \cdot \mathrm{C}_{6} \mathrm{H}_{4} \mathrm{NO}_{2}\right)_{2}$.

$$
\begin{array}{cccc}
\text { Procente: N } & 4.88, & \text { Cl } 24.74 . \\
\text { Gef. } & \$ & 5.34, & 25.30 .
\end{array}
$$

Tetrachlorphtalsaurer Cetylester.

a) Aus tetrachlorphtalsaurem Silber und Cetyljodid.

Die berechneten Mengen der beiden Körper wurden 2-3 Stunden auf dem Wasserbade erwärmt, das Reactionsproduct mit Essigăther extrahirt und die Lösung mit Methylalkohol gefällt. Der Ester fällt dann in Blättchen aus. Durch Wiederholen der Operation wird er rein erhalten. Schmp. $49-50^{\circ}$.

Die Ausbeute ist naheza quantitativ.

Bei der Verseifung wurde Tetrachlorphtalsäure und Cetylalkobol erhalten.

Analyse: Ber. für $\mathrm{C}_{6} \mathrm{Cl}_{4}\left(\mathrm{COO} \mathrm{C}_{16} \mathrm{H}_{33}\right)_{2}$.

Procente: Cl 18.88.

Gef. $\gg \quad \gg 19.45,19.10$. 
b) Aus Tetrachlorphtalylchlorid und Cetylalkohol.

Natriumcetylat und Tetrachlorphtalylchlorid werden in berechneter Menge gemischt und auf dem Wasserbade erwärmt, bis der Geruch des Chlorides fast verschwunden ist, darauf mit Natronlauge und Aether behandelt. Der rohe Cetylester wird wieder durch Lösen in Essigăther und Fällen mit Holzgeist rein erhalten. Er war mit dem ans dem Silbersalz und Cetyljodid erhaltenen identisch. Schmp. $49-50^{\circ}$.

Die Ausbeute war auch in diesem Falle gering.

Analyse: Ber. für $\mathrm{C}_{6} \mathrm{Cl}_{6}\left(\mathrm{COOC}_{68} \mathrm{H}_{33}\right)_{2}$.

$$
\text { Gef. Procente: Ol 18.88. }
$$

Durch die mitgetheilten Versuche sind die folgenden Ester der Phtalsäure und Tetrachlorphtalsäure dargestellt worden:

Nentraler phtalsaurer Benzylester. . . . . Schmp. 42-430

> $\quad p$-Nitrobenzylester . . \ $154-155^{\circ}$

, Cetylester . . . . . . , 42-430

- tetrachlorphtalsaurer Benzylester . . > $92-93^{0}$

, $p$-Nitrobenzylester $\quad 179-180^{\circ}$

\ Cetylester . . \ $49-50^{\circ}$

Saurer phtalsaurer Benzylester . . . : . . , 102-1040

- tetrachlorphtalsaurer Benzylester . . . , $130-131^{0}$

Die mittels der Silbersalze einerseits, mittels der Chloride andererseits dargestellten neutralen Ester haben sich in allen Fällen als völlig identisch erwiesen; insbesondere zeigten die entsprechenden Präparate jedesmal genau den gleichen Schmelzpunkt.

Da in der Umsetzung der Silbersalze mit Alkylhalogeniden eine Umlagerung kaum anzunehmen ist, die Chloride der Phtalsäure und Chlorphtalsäure aber nach den meisten ihrer Reactionen unsymmetrisch gebant erscheinen, so bleibt wohl nichts anderes übrig, als den Estern symmetrische Constitution zuzuschreiben, und zu folgern, dass die Umlagernng bei der Umsetzung der Chloride mit den Alkoholen stattfindet. Man kann auch die Chloride als tautomere Körper auffassen.

Bei dem Phtalylchlorid wäre es noch denkbar, dass der flüssige Körper eine Mischung des symmetrischen und des unsymmetrischen Chlorids darstellt ${ }^{1}$ ). In diesem Falle wäre aber bei der Umsetzung mit den Alkoholen das gleichzeitige Auftreten isomerer Ester zu erwarten, was in keinem Falle beobachtet wurde. Auch wäre

1) Ist es ja kürzlich I. Remsen gelungen, 2 isomere Chloride der o-Sulfobenz oēsāure zu erhalten (diese Berichte 29, Ref. 352 ff; Amer. Chem. Journ. 18, 791. 
diese Hypothese auf das Chlorid der Tetrachlorphtalatiare nicht anwendbar, da dieses fest und darch einen scharfen Schmelepunt elmaralkterisirt ist.

Schliesslich sei noch einmal anf die merkwördige Umeetzumg zwischen tetrachlorphtaloaurem Silber and $p$-Nitrobenzyljodid hingewiesen, welche spontan zwischen diesen beiden festen Kotpern vor sich geht and ohne irgend welche Verflüssigning $2 n$ Ende geführt werden kann.

Braunschweig, Techn. Hochschule, Laboratorium für analyt. und techn. Chemie.

Berich tigungen.

Jahrgang 29, Heft 19 (Register), S. 1262, Z. 20 v. o. ist nach der Seitanangabe 32640 ein Absatz zu machen und vor die folgenden Worte einzuschieben: «Meyer, V.«

» 29, " 19 (Register), S. 1292 ist vor dem Buchstaben $Z$ einzaschieben: Y. Young, George: Synthese des Diphenyloxytriazols $R$ 85; Substituirte Phenyloxytriazole 22311.

"30, 4 S. 398, Z. 10 v. o. lies : "Das Safranol e statt -Dasselber. 